\title{
Aide au suicide - à propos de l'attitude adoptée dans l'accompagnement
}

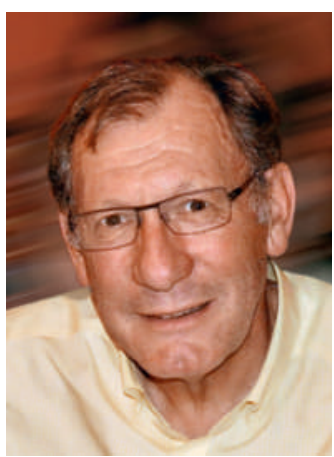

Jean Martin
Dès le début des années 1990, j'ai été concerné par la problématique de l'assistance au suicide. Elle était alors à ses débuts dans des situations médicalisées - aide à des personnes gravement atteintes dans leur santé et j'ai eu à me pencher sur les aspects médico-légaux et médico-éthiques (ai présenté ma position dans des ouvrages collectifs $[1,2])$. La Commission nationale d'éthique a établi deux rapports à son propos [3, 4].

Aujourd'hui le sujet est devenu très visible et retient l'attention au plan national et international, notamment par les associations Exit (Deutschschweiz et Suisse romande) et Dignitas; rappelons par exemple les deux votations zurichoises de 2011, qui ont refusé des limites plus contraignantes à cette pratique.

Il y a quelques mois, sachant qu'un confrère estimé, ancien chef de service de médecine interne, était accompagnant Exit, j'ai souhaité le rencontrer (N.B.: je ne suis pas membre d'Exit, tout en ayant eu des contacts avec ses responsables dans ma fonction antérieure). S'agissant d'un entretien avec un seul intervenant, ce qui suit n'a pas de prétention à être représentatif. Je juge utile de le décrire dans la mesure où on penserait trouver, parmi les collaborateurs d'Exit, surtout des personnalités militantes, «pour la cause». Ce n'est pas forcément le cas.

Propos de ce médecin: «Je n'avais pas d'intérêt particulier pour l'assistance au suicide mais, sur demande d'un ami français dans une situation critique, j'ai pris des informations auprès d'Exit Suisse romande et on m'a proposé d'assister à ce qu'ils appellent auto-délivrance. J’y suis allé et ai été impressionné par le déroulement de la dite assistance, notamment par la clarté de la situation et la sérénité ambiante, qu'il s'agisse du malade, des proches, des échanges entre les personnes présentes.»

Ce confrère s'est alors mis à disposition de l'association. Au cours de cinq ans, il a accompagné 25 suicides. Cette expérience a été enrichissante (mais absolument pas au plan pécuniaire, bien entendu) et il est prêt à poursuivre. Cela étant, d'une manière à laquelle je ne m'attendais pas, il refuse explicitement toute militance. Il fait ces accompagnements parce qu'estimant, en conformité avec la situation légale, qu'on doit pouvoir recevoir une aide en vue de mettre un terme à une existence très lourde et sans perspective d'amélioration. Il entend ainsi rendre le service d'un humain à un autre humain (civiquement, solidairement pourrait-on dire); permettant ainsi à une personne souffrant gravement de prendre de manière autonome une décision concernant son existence.
Au cours d'un premier entretien, il s'assure des caractéristiques de la demande, prenant notamment connaissance du dossier médical; il se convainc de la volonté claire et répétée du patient et du fait que ces éléments rendent compréhensible la décision de mourir. Il le fait, me dit-il, en restant neutre, sans implication affective ni geste ou parole qui indiquerait qu'il trouve bonne cette décision. A quelques reprises, il a refusé d'intervenir parce que la situation n'emportait pas l'adhésion.

Lors de l'accompagnement du suicide quelques semaines plus tard, il décrit un rôle parfaitement courtois mais gardant une distance: vérifiant que la détermination du malade reste inchangée et entière, là aussi sans manifestation qui pourrait être vue comme un encouragement. En termes simples: il se met à disposition pour remettre à la personne une dose létale de pentobarbital qu'il a prescrite et lui tend le verre d'eau qui lui permet de l'avaler.

Chose qui mérite d'être relevée, à chaque fois il ressent un inconfort quand il s'en va au domicile du malade pour l'accompagner dans le suicide, et cet inconfort reste avec lui au retour. Il le dit en toute franchise et je le rapporte comme tel - en sachant qu'on pourrait y voir la traduction de ce que la démarche est discutable voire condamnable. Toutefois il est possible d'y voir un signe du caractère correct de ce qui se passe. En effet, on peut penser que l'assistance au suicide doit garder une dimension de transgression, acceptable cependant dans des situations données.

La position ci-dessus me paraît judicieuse: répondre, sans l'influencer, à la libre détermination du patient complètement informé et capable de discernement qui présente une situation faite de souffrance, de dépendance et d'irréversibilité. Dans le sens d'un échange, d'une collaboration entre deux personnes libres chacune de leurs faits et gestes - et sans aucun lien entre elles qui pourrait donner lieu à conflit d'intérêt. Ceci dans un cadre privé, l'Etat n'ayant pour rôle que de surveiller que ce qui est fait ne nuit pas à autrui - et un contrôle a posteriori par l'Ordre judiciaire. Etant entendu aussi qu'il importe que de leur côté les pouvoirs publics évitent toute implication qui pourrait être vue comme une validation du suicide.

Jean Martin, ancien médecin cantonal

et membre de la rédaction 